\title{
A TWO-STEP TRANSSHIPMENT MODEL WITH FUZZY DEMANDS AND SERVICE LEVEL CONSTRAINTS
}

\author{
Ben Said, L.; Hmiden, M. \& Ghedira, K. \\ Unité de Recherche en Stratégie d’Optimisation des Informations et de la connaissancE (SOIE), \\ ISG de Tunis 41 avenue de la liberté cité Bouchoucha 2000 Tunis, Tunisia \\ E-Mail: lamjed.bensaid@isg.rnu.tn,mohamed.hmiden@gmail.com,khaled.ghedira@isg.rnu.tn
}

\begin{abstract}
We consider a distribution network composed of one supplier and several non-identical locations characterized by fuzzy customer demands and service level constraints. These locations could cooperate together via product transfer known as transshipment. The transshipment problem consists in determining the replenishment quantities that minimize the total inventory cost where a specific transfer policy is practiced. Our objectives in this paper are to identify an inter-location transfer policy that participates to satisfy the service level constraints and to determine the approximate replenishment quantities. To achieve these objectives we propose: (1) a new transshipment model based on the chance constrained programming, (2) a two-step transshipment policy that differs from classic ones by product transfer from locations in need to others also in need and (3) a hybrid algorithm based on fuzzy simulation and genetic algorithms to approximate replenishment quantities.

(Received in March 2009, accepted in September 2009. This paper was with the authors 2 months for 1 revision.)
\end{abstract}

Key Words: Transshipment Problem, Service Level Constraints, Fuzzy Customer Demands, Fuzzy Simulation, Genetic Algorithm

\section{INTRODUCTION}

The supply chain is a network of suppliers, manufacturers, distributors and consumers. It ensures mainly two types of functions: (1) physical functions consisting in product transformation, storage and transportation and (2) mediation functions consisting in matching demand and supply in a dynamic and uncertain environment [1]. The supply chain managers' aim is to ensure a good trade-off between cost and realized service level. This trade-off depends on the nature of customer demands. In fact, Fisher [1] identified mainly two product classes: functional and innovative products. The first class satisfies customer basic needs. It includes products recognised by predictable and stable demands, a long life cycle and low profit margin. However, the second class includes products characterised by a short life cycle and a high profit margin. In order to ensure the trade-off between costs and service level, several methods could be adopted such as collaborative inventory management known as transshipment [2]. This method allows the regulation of the inventory level to face the unexpected customer demand fluctuation. A reduced inventory level causes the non satisfaction of customer requirements and an inventory excess generates holding costs. This task becomes more complex when it concerns several locations. Transshipment is widely used in practice to reduce inventory costs and to improve the customer service level [3]. It provides an effective mechanism for correcting discrepancies between the locations observed customer demands and their available inventories [4].

The transshipment problem consists in determining the replenishment quantities where a specific transfer policy is practiced [5]. This problem has been largely studied where several configurations and parameters were considered. Literature identifies mainly two transshipment configurations [6]: (1) emergency transshipment where the product transfer is 
made at the end of the period after observing the customer demands and (2) preventive transshipment where the product redistribution is realized at the beginning of the period [7]. Concerning the transshipment problem parameters, we can identify mainly the three following categories: (a) replenishment parameters such as the replenishment lead times [8] and the replenishment fixed cost [9], (b) transfer parameters such as the transshipment mean capacity [10] and the transshipment lead times [11] and (c) environment parameters such as the number of locations [12], the number of periods [6] and the customer demands [7, 12, 8, 5]. The only uncertain parameter considered in these works is the customer demands. Generally, the latter is modelled by probabilistic approach based on historical data analysis [13]. However, these data are not always available especially for innovative products having a short life cycle [14]. In this case, fuzzy sets could be adopted to model the customer demands uncertainties and the use of fuzzy demand simulation instead of stochastic demand and standard simulation can be considered the most suitable and efficient method to study our transshipment problem.

Few researches applied the fuzzy sets to model the uncertainty of the different inventory parameters. For example $[15,16]$ have studied the inventory problem with fuzzy replenishment lead time, [16, 17] have considered fuzzy inventory costs and [18-21] studied inventory management with fuzzy customer demands.

In competitive markets, customers become seriously more sensitive to the service quality especially for innovative products. Indeed, product shortages significantly affect companies' reputation. Consequently, in order to persist in a turbulent market and achieve high profit margins besides the inventory costs reduction, the service level improvement became a vital objective [18]. Several researches have considered inventory optimization problems with service level constraints [22-25]. In particular, Young et al. [26] proposed a transfer policy integrating the emergency and preventive transshipment to adjust the service level.

The contribution of our work in this paper is threefold. First, we represent customer demands by fuzzy sets. In addition, we incorporate location service level constraint to the transshipment problem and we propose a new model for this problem based on chance constrained programming. Second, we propose a new transshipment policy characterized by the transfer from locations in need to others in need. Third, we propose a hybrid algorithm based on fuzzy simulation and genetic algorithm to approximate replenishment quantities.

The remainder of this paper is organized as follows: in section two, we present the studied transshipment problem. In section three, we introduce the proposed transshipment policy. In section four, we present the transshipment problem model and the hybrid algorithm developed to approximate the optimal replenishment quantities. In section five, we discuss the experimental results and we finish with a conclusion.

\section{PROBLEM DESCRIPTION}

In our work, we consider the transshipment problem characterized by $N$ locations having nonidentical cost structures and uncertain customer demands. These locations are periodically reviewed and during each period the following events are sequentially executed (Fig. 1): (1) at the beginning of the period, each location $L_{i}(i \in\{1, \ldots, N\})$ launches a replenishment order to receive the quantity $S_{i}$, we assume that the replenishment lead time is negligible, (2) during the period, each location $L_{i}$ observes its customer demands and (3) at the end of the period, each location $L_{i}$ evaluates its realized service level, the transshipment operations are triggered and the customer demands are partially or entirely satisfied. This sequence of events differs from that adopted by Herer et al. [24] by considering the realized service level. In fact, we focus on a distribution system where each location $L_{i}$ has to realize at least a minimum service level equals to $\beta_{i}$. 


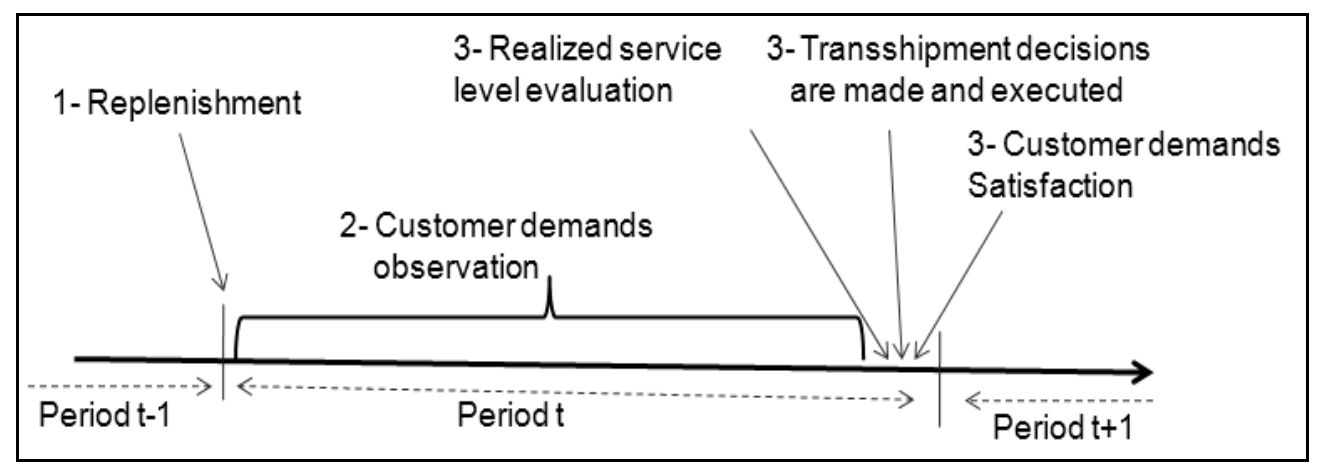

Figure 1: Sequence of events during a period.

\subsection{Uncertain customer demands}

For innovative products characterized by short life cycle, the customer demands are generally estimated referring to manager judgments witch are often expressed by imprecise linguistic expressions such as "customer demands at our location is most likely to vary between b and $c$ units" or "customer demands at our location will be about b units". Fuzzy sets are found to be useful in representing these approximate qualifiers, due to their conceptual and computational simplicity [27]. In fact, the above mentioned expressions could be represented respectively by trapezoidal and triangular fuzzy sets as shown in Fig. 2.

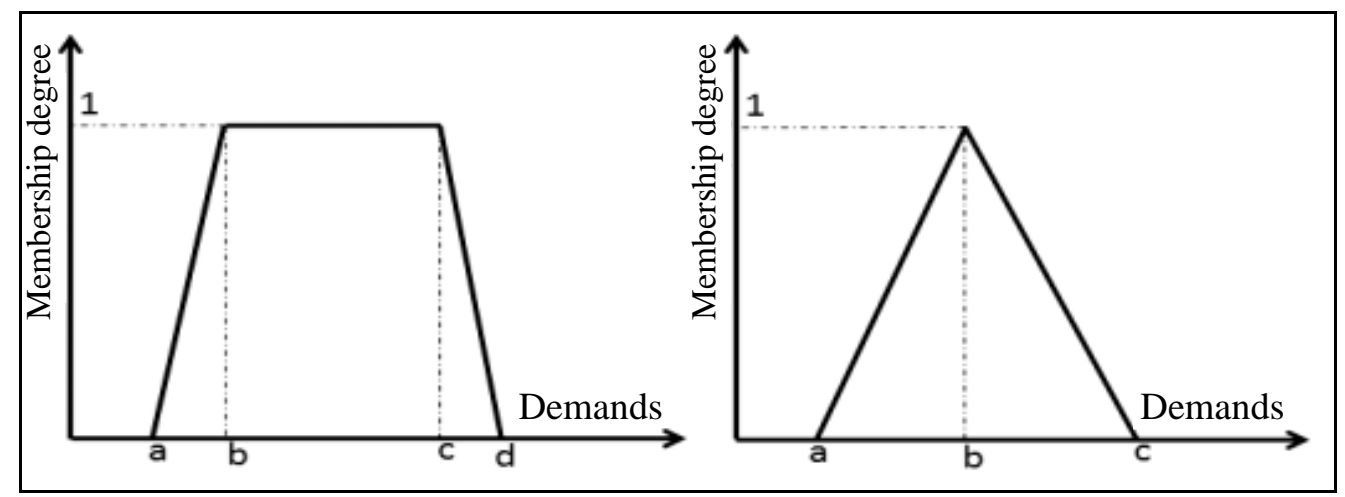

Figure 2: Trapezoidal and triangular fuzzy demands.

In this work, we adopt the trapezoidal fuzzy set representation to model the customer demands.

\subsection{Notations and fuzzy concepts}

Throughout this paper, we adopt the following notations concerning decision variables, system parameters and fuzzy concepts:

Decision variables:

- $S=\left(S_{1}, S_{2}, \ldots, S_{N}\right)$ - vector of replenishment quantities, where $i \in\{1, \ldots, N\}$ and $S_{i}$ represents the replenishment quantity at the location $L_{i}$

- $\quad T_{i j}$ - transhipped quantity from the location $L_{i}$ to the location $L_{j}$

System parameters:

- $C_{i}$ - unit replenishment cost at the location $L_{i}$

- $H_{i}$ - unit holding cost at the location $L_{i}$

- $C_{i, j}$ - unit transshipment cost from location $L_{i}$ to location $L_{j}$. We assume that this cost is symmetric $\left(C_{i, j}=C_{j, i}\right)$. We assume also that $H_{i} \geq C_{i, j}$ 
- $\beta_{i}$ - the minimal service level for the location where $L_{i}, \beta_{i} \in[0,1]$

- $R S L_{i}$ - the realized service level for location $L_{i}$ where $R S L_{i} \in[0,1]$

- $D=\left(D_{1}, D_{2}, \ldots, D_{N}\right)$ - vector of fuzzy customer demands, where $D_{i}$ represents the fuzzy customer demand at the location $L_{i}$. $D_{i}$ is modelled by a trapezoidal fuzzy sets defined by the quadruplet $\left(a_{i}, b_{i}, c_{i}, d_{i}\right)$

Fuzzy concepts:

- $\mu(D)=\min \left(\mu_{1}\left(D_{1}\right), \mu_{2}\left(D_{2}\right), \ldots, \mu_{\mathrm{N}}\left(D_{N}\right)\right)$, where $\mu_{\mathrm{i}}$ represents the membership function of the fuzzy customer demand at the location $L_{i}$

- $\operatorname{Pos}\left(D_{i} \geq r\right)=\sup \mathrm{x} \geq \mathrm{r} \mu_{\mathrm{i}}(\mathrm{X})$ - the possibility degree that the fuzzy customer demand in location $L_{\mathrm{i}}$ is greater than a given value $r$. The fuzzy event could fail even if the value returned by this function is equal to 1

- $\operatorname{Nec}\left(D_{i} \geq r\right)=1-\sup _{\mathrm{X} \leq \mathrm{r}} \mu_{\mathrm{i}}(\mathrm{X})$ - the necessity degree that the fuzzy demand at location the $L_{i}$ is greater than a given value $r$

\section{PROPOSED TRANSSHIPMENT POLICY}

We assume that for each location $L_{i}$ the realized service level, at the end of the period, must be superior or equal to a minimum service level $\beta_{i}$. Consequently, for each location we have a service level constraint. To satisfy these constraints, we propose a new transshipment policy that differs from those proposed in previous researches. In fact, our policy is characterized by a two-step transshipment.

\subsection{First step: excess-to-need transshipment}

In this step, the transshipment is executed from locations in excess to locations in need. This step is divided into two phases described bellow.

\subsubsection{First phase}

The objective of this phase consists in improving the realized service level for locations having an unsatisfied service level constraint. In order to achieve this objective, the product transfer is executed from $L_{i}$, $i$ belongs to the set $I^{+}$of sending locations in excess, to $L_{j}, j$ belongs to the set $J$ of receiving locations in need and accomplishing realised service level $R S L_{j}$ less that $\beta_{j}$. The transhipped quantities $X_{i j}$ are determined by the resolution of the following linear program:

$$
\max \sum_{i \in I^{+}} \sum_{j \in J^{-}}\left(H_{i}-C_{i j}\right) * X_{i j}
$$

Subject to:

$$
\begin{gathered}
\sum_{\mathrm{j} \in \mathrm{J}^{-}} \mathrm{X}_{\mathrm{ij}} \leq \mathrm{S}_{\mathrm{i}}-\mathrm{D}_{\mathrm{i}}, \forall \mathrm{i} \in \mathrm{I}^{+} \\
\sum_{\mathrm{i} \in \mathrm{I}^{+}} \mathrm{X}_{\mathrm{ij}} \leq \mathrm{D}_{\mathrm{j}}^{*}\left(\beta_{\mathrm{j}}-\mathrm{RSL}_{\mathrm{j}}\right), \forall \mathrm{j} \in \mathrm{J}^{-} \\
\mathrm{X}_{\mathrm{ij}} \geq 0
\end{gathered}
$$

The objective function - Eq. (1) consists in maximizing the profit achieved by the transshipment. In fact, for each product unit transhipped from $L_{i}$ to $L_{j}$, the achieved profit is evaluated to $\left(H_{i}-C_{i j}\right)$. The set of constraints - Eq. (2) concerns locations in excess and restricting the total transhipped quantity from location $L_{i}$ to its excess inventory $\left(S_{i}-D_{i}\right)$. The set of constraints - Eq. (3) is relative to locations having unsatisfied service level limiting the 
total transhipped quantity towards $L_{j}$ to its required quantity in order to bring its realized service level from $R S L_{j}$ to $\beta_{j}$.

Once this phase is executed, two situations are possible: (1) locations having unsatisfied service level constraint still exist. In this case, we apply the second step of our transshipment policy or (2) all the service level constraints are satisfied and there are locations in need and others in excess. In this case, we apply the second phase of this first step.

\subsubsection{Second phase}

The aim of this phase is to reduce the total holding cost once the service level constraints have been already satisfied during the first phase. The transshipment is from locations in excess belonging to the set of locations noted $I_{1}{ }^{+}$to locations in need and having a satisfied service level constraint belonging to the set of locations noted $J_{1}$. The transhipped quantities $Y_{i j}$ between locations during this phase could be obtained by resolving the following linear program:

$$
\max \sum_{i \in I_{1}^{+}} \sum_{j \in J_{1}^{-}}\left(H_{i}-C_{i j}\right) * Y_{i j}
$$

Subject to:

$$
\begin{gathered}
\sum_{j \in J_{1}^{-}} Y_{i j} \leq S_{i}-\left(D_{i}+\sum_{j \in J^{-}} X_{i j}\right), \forall i \in I_{1}^{+} \\
\sum_{i \in I_{1}{ }^{+}} Y_{i j} \leq D_{j}-\left(S_{j}+\sum_{i \in I^{+}} X_{i j}\right), \forall j \in J_{1}^{-} \\
Y_{i j} \geq 0
\end{gathered}
$$

The constraints relative to the objective function - Eq. (4) are classified into two categories: the first one - Eq. (5) is relative to locations in need indicating that the quantities transhipped to these locations should not exceed their required quantity necessary to bring their realized service level to 1 . The second constraint - Eq. (6) is relative to locations in excess indicating that quantities transhipped from these locations should not exceed their surplus quantity.

\subsection{Second step: need-to-need transshipment}

This step is executed only if the distribution network satisfies simultaneously the two following conditions: (C1) all locations of the distribution network are in need, (C2): there exist locations having satisfied and others unsatisfied service level constraints. In this step, the transshipment is executed from locations in need to others also in need. In fact, we tranship from the set of locations having a satisfied service level constraint, noted $I$, to the set of locations having unsatisfied service level constraint, noted $J_{2}$, a subset of $J_{1}$.

We allow transshipment from locations $L_{i}$ to $L_{j}$ by cancelling the last observed demands once the service level constraint is satisfied for the sending locations. The transhipped quantities $Z_{i j}$ during this step are determined by resolving the following linear program:

$$
\min \sum_{i \in I^{-}} \sum_{j \in J_{2}^{-}} C_{i j} * Z_{i j}
$$

Subject to:

$$
\begin{aligned}
& \sum_{i \in I^{-}} Z_{i j}=D_{j}^{*}\left(\beta_{j}-R S L_{j}\right), \forall j \in J_{1}^{-} \\
& \sum_{j \in J_{2}^{-}} Z_{i j} \leq D_{i}^{*}\left(R S L_{i}-\beta_{i}\right), \forall i \in I^{-}
\end{aligned}
$$


The set of constraints - Eq. (8) is relative to locations belonging to $J_{1}{ }^{-}$and having unsatisfied service level constraint restricting the total transhipped quantity to that necessary to bring the realized service level from $R S L_{j}$ to $\beta_{j}$. The second category of constraints - Eq. (9) is relative to locations in need and satisfying their service level constraint. Each one limits the total transhipped quantity from these locations to their surplus quantity once their service level constraint is satisfied.

The total transhipped quantity $T_{i j}$ from location $L_{i}$ to $L_{j}$ is equal to sum transhipped quantities during the first and the second steps - Eq. (10) and the total transshipment cost is equal to $K$ - Eq. (11).

$$
\begin{gathered}
T_{i j}=X_{i j}+Y_{i j}+Z_{i j} \\
K=\sum_{i=1}^{N} \sum_{j=1, j \neq i}^{N} C_{i j} * T_{i j}
\end{gathered}
$$

Fig. 3 illustrates an example of our proposed two-step transshipment policy execution for a distribution network composed of 7 locations.

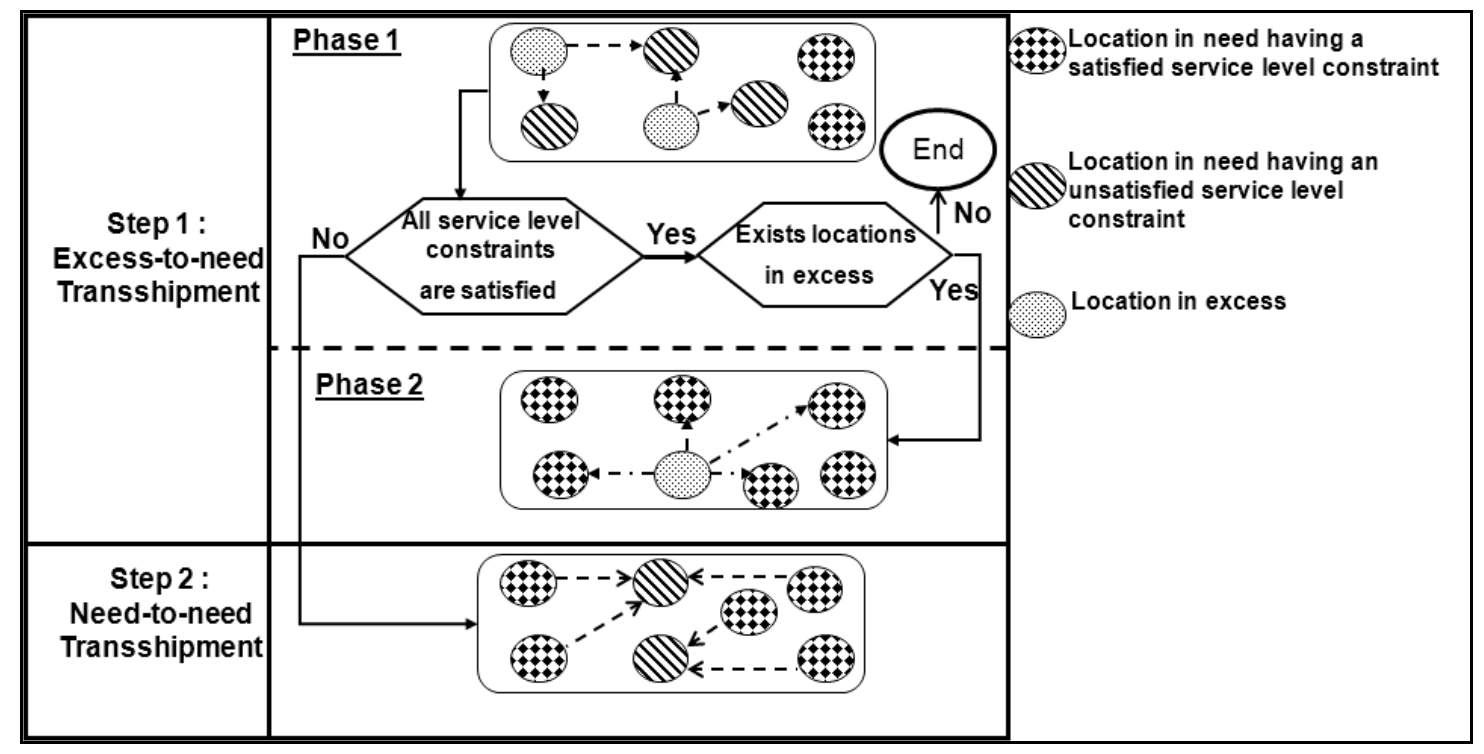

Figure 3: Two-step transshipment policy illustration.

\section{REPLENISHMENT QUANTITIES}

In this section, we aim to determine the approximate replenishment quantities while the above described transshipment policy is practiced. To achieve this objective, we propose: (1) a new formalisation of the transshipment problem and (2) a hybrid algorithm based on fuzzy simulation and genetic algorithm.

\subsection{Model description}

We are interested in fulfilling the service level constraints under the minimum inventory costs. The total inventory cost function $\mathrm{C}(S, D)$ considered here is composed of the replenishment cost, the holding cost and the transshipment cost. We ignore the shortage cost since the service level constraints are considered [22]. In fact, for each location $L_{i}$, we have a service level constraint indicating that the realized service level $\left(R S L_{i}\right)$ should be greater or equal to its predefined minimum service level $\beta_{i}$. 
Subject to:

$$
C(S, D)=\sum_{i=1}^{N} C_{i} * S_{i}+\sum_{i=1}^{N} H_{i} * \max \left(0, S_{i}-D_{i}\right)+K
$$

$$
R S L_{i} \geq \beta_{i}, i=1, . ., N
$$

$R S L_{i}$ is given by Eq. (14). It is the fraction of customer demands met by inventory on hand (IO) at the end of the period. It is equal to 1 if the inventory on hand, given by (15), exceeds the demand $D_{i}$ otherwise it is equal to the inventory on hand divided by demands.

$$
\begin{aligned}
R S L_{i} & =\left\{\begin{array}{cc}
1 & D_{i} \leq I O_{i} \\
I O_{i} / D_{i} & D_{i} \geq I O_{i}
\end{array}\right. \\
I O_{i} & =S_{i}+\sum_{j=1, j \neq i}^{N} T_{j i}-\sum_{j=1, j \neq i}^{N} T_{i j}
\end{aligned}
$$

Since we are interested in satisfying the service level constraints under minimum inventory costs, our transshipment problem could be formulated by the following linear program:

$$
\begin{gathered}
\min C(S, D) \\
R S L_{i} \geq \beta_{i} \quad i=1, \ldots, N
\end{gathered}
$$

Given that the demand is fuzzy, the total inventory costs $C(S, D)$ and the service level constraints are imprecise. (16) and (13) could be modelled by fuzzy numbers whose membership functions are unknown since they depend on the decision vector $S$ of replenishment quantities and the fuzzy vector $D$. We can verify for a particular vector $S^{0}$ if the service level constraints are satisfied. Thus, $S^{0}$ is feasible solution if the possibility or the necessity measure of the fuzzy events $\left(R S L_{i} \geq \beta_{i}\right)$ exceeds a predefined level $\alpha$.

Since $C(S, D)$ is not well defined, we can search for the minimum value $T$ so that $C(S, D)$ $\leq T$. As the event $C(S, D) \leq T$ is not well defined, we can measure its possibility in an optimistic sense or its necessity in pessimistic sense. If the possibility or the necessity measure exceeds some predefined confidence level $\theta, T$ is considered as the optimistic or pessimistic total inventory cost. Since $D$ is a fuzzy vector, we can convert the (16) and (13) to the following fuzzy chance constrained program.

$$
\left\{\begin{array}{c}
\min T \\
\operatorname{Pos}[C(S, D) \leq T] \quad \geq \theta \\
\operatorname{Pos}\left[R S L_{i} \geq \beta_{i}\right] \geq \alpha
\end{array}\right.
$$

\subsection{Proposed hybrid algorithm}

To determine the optimal solution of the decision vector $S=\left(S_{1}, S_{2}, \ldots, S_{N}\right)$, we propose a hybrid algorithm based on: (1) fuzzy simulation to verify the feasibility of a particular solution and to determine its total inventory cost and (2) genetic algorithm to derive the approximate solution.

\subsubsection{Fuzzy simulation}

Liu and Iwamura [28] proposed a fuzzy simulation process to check the feasibility of a particular solution relative to a fuzzy chance constrained program (18). We adapt this process to verify if a particular decision vector $\mathrm{S}^{\Delta}$ satisfies the service level constraints. The process steps are presented in the algorithm IsFeasible witch checks if $\operatorname{Pos}\left(R S L_{i} \geq \beta_{i}\right) \geq \alpha$ for each location $L_{i}$.

To determine the minimum value of the total inventory cost $T^{*}$ for a particular solution $S^{4}$, we distinguish two strategies: 
- Optimistic strategy: we search for a particular generated customer demand instance that satisfies the constraint $C\left(S^{4}, D\right) \leq T^{*}$. In this strategy, we refer to the possibility measure to check this constraint and we use the MinCostOpt algorithm in Fig. 5 (a) to derive $T^{*}$ such that $\operatorname{Pos}\left[C\left(S^{4}, D\right) \leq T^{*}\right] \geq \theta$.

- Pessimistic strategy: we derive $T^{*}$ such that all the generated customer demands satisfy the constraint $C\left(S^{4}, D^{k}\right) \leq T^{*}, k=1, \ldots, m$, where $m$ is the number of simulation step. We use the MinCostPest algorithm illustrated in Fig. 5 (b) to calculate $T^{*}$ such that $\operatorname{Nec}\left[C\left(S^{4}, D\right) \leq T^{*}\right] \geq$ $\theta$.

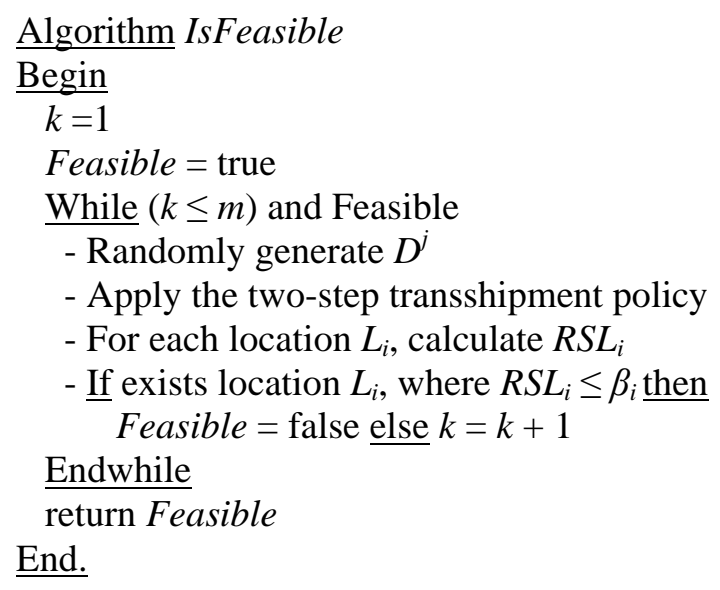

Figure 4: IsFeasible algorithm.

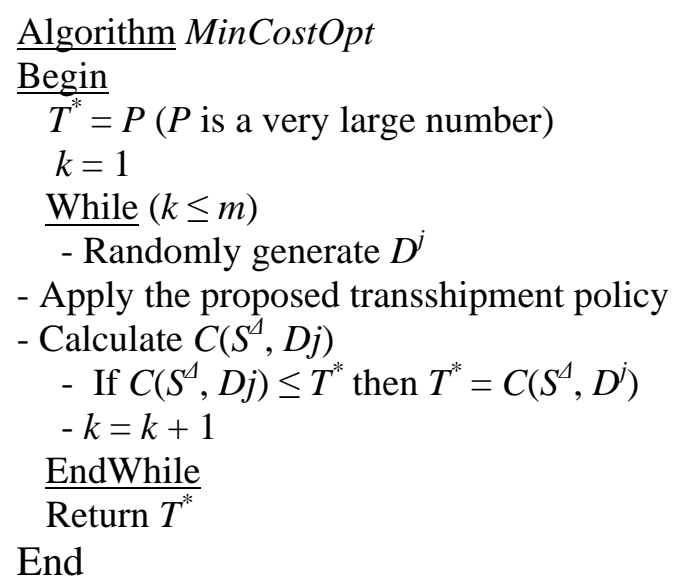

- Apply the proposed transshipment policy

- Calculate $C\left(S^{4}, D j\right)$

- If $C\left(S^{4}, D j\right) \leq T^{*}$ then $T^{*}=C\left(S^{4}, D^{j}\right)$

$-k=k+1$

EndWhile

Return $T^{*}$

End

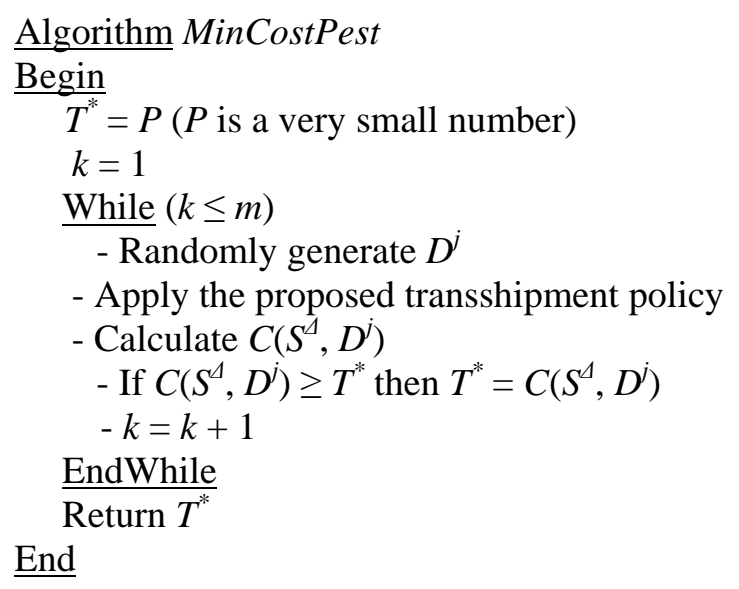

(b)

Figure 5: (a) MinCostOpt algorithm, (b) MinCostPest algorithm.

\subsubsection{Genetic algorithm}

Genetic algorithms are search methodology invented by Holland [29] and inspired by the natural genetic theory. Our genetic algorithm is characterized by:

- Coding: chromosomes represent replenishment vector where each gene codes the replenishment quantity for a particular location. The genes are real coded.

- Initialization: we use a population of 30 randomly generated individuals. These individual are only added to the population if their feasibility is proved using the fuzzy simulation (algorithm IsFeasible illustrated in Fig. 4). 
- Evaluation: it consists in determining the fitness value for each individual. In our study, the fitness represents the total inventory cost value. We refer to fuzzy simulation (MinCostOpt or MinCostPes algorithms, respectively Figs. 5 (a) \& 5 (b)) to calculate the fitness. Once this operation is achieved, we sort ascending the population individuals according to their fitness.

- Selection: in our case, the best individuals represent the set of replenishment decisions $\left\{S^{*}\right\}$ that ensure low total inventory cost. The roulette wheel selection is used to choose the individuals that form the next generation. In addition, the best $10 \%$ individuals are directly copied to integrate the next generation.

- Crossover: binary tournament selection is used to pick out parents for reproduction. Tournament selection runs a tournament between two randomly chosen individuals and selects the winner (individual with best fitness value). A two-point crossover operator is applied in order to reproduce children that are added to the new generation only if their feasibility is proved.

- Mutation: this operation consists in mutating an individual by modifying one randomly selected gene. The feasibility of the mutated individual is added to the new generation if and only if its feasibility is proved.

\section{ILLUSTRATIVE CASE}

In order to evaluate our transshipment policy, we illustrate the case of a distribution network formed by four locations and selling a fashion product characterized by a short life cycle. These locations are supplied at the beginning of a period by the one supplier. To keep a good reputation and to persist in the turbulent market, locations have to achieve a good service level. For each location, the customer demands are modelled by trapezoidal fuzzy and minimal service level constraint is considered. The distribution network locations customer demands, inventory costs and transshipment costs are represented in the following Table I.

Table I: Distribution network locations characteristics.

\begin{tabular}{|c|c|c|c|c|}
\hline $\begin{array}{c}\text { Location } \\
L_{i}\end{array}$ & $\begin{array}{c}\text { Fuzzy demands } \\
\left(a_{i}, b_{i}, c_{i}, d_{i}\right)\end{array}$ & $\begin{array}{c}\text { Holding cost } \\
H_{i}\end{array}$ & $\begin{array}{c}\text { Replenishment cost } \\
C_{i}\end{array}$ & $\begin{array}{c}\text { Transshipment cost } \\
C_{i, j}\end{array}$ \\
\hline$L_{1}$ & $(5,8,17,20)$ & 7 & 10 & $C_{1,2}=6, C_{1,3}=6, C_{1,4}=5$ \\
\hline$L_{2}$ & $(3,6,15,18)$ & 8 & 15 & $C_{2,1}=6, C_{2,3}=5, C_{2,4}=5$ \\
\hline$L_{3}$ & $(8,12,17,20)$ & 7 & 13 & $C_{3,1}=6, C_{3,2}=5, C_{3,4}=6$ \\
\hline$L_{4}$ & $(6,8,15,18)$ & 9 & 12 & $C_{4,1}=5, C_{4,2}=5, C_{4,3}=6$ \\
\hline
\end{tabular}

\subsection{Comparing transshipment policies}

To verify the efficiency of our transshipment policy, we compare between three system configurations: (1) inventory management without transshipment, (2) inventory management where the classical transshipment for locations in excess to locations in need is practiced [23] and (3) inventory management where our transshipment policy is practiced. The comparison criteria are the total inventory cost and the total replenishment quantity.

In this experimentation, we choose a common minimum service level $\beta$ for all locations equal to 0.80 and we are interested in demands having a membership degree greater or equal to 0.80. As shown in Fig. 6 (a) the lowest total inventory cost is obtained when our transshipment policy is practiced. This result can be explained by the fact that our transshipment policy provides the lowest total replenishment quantity as shown in Fig. 6 (b). 


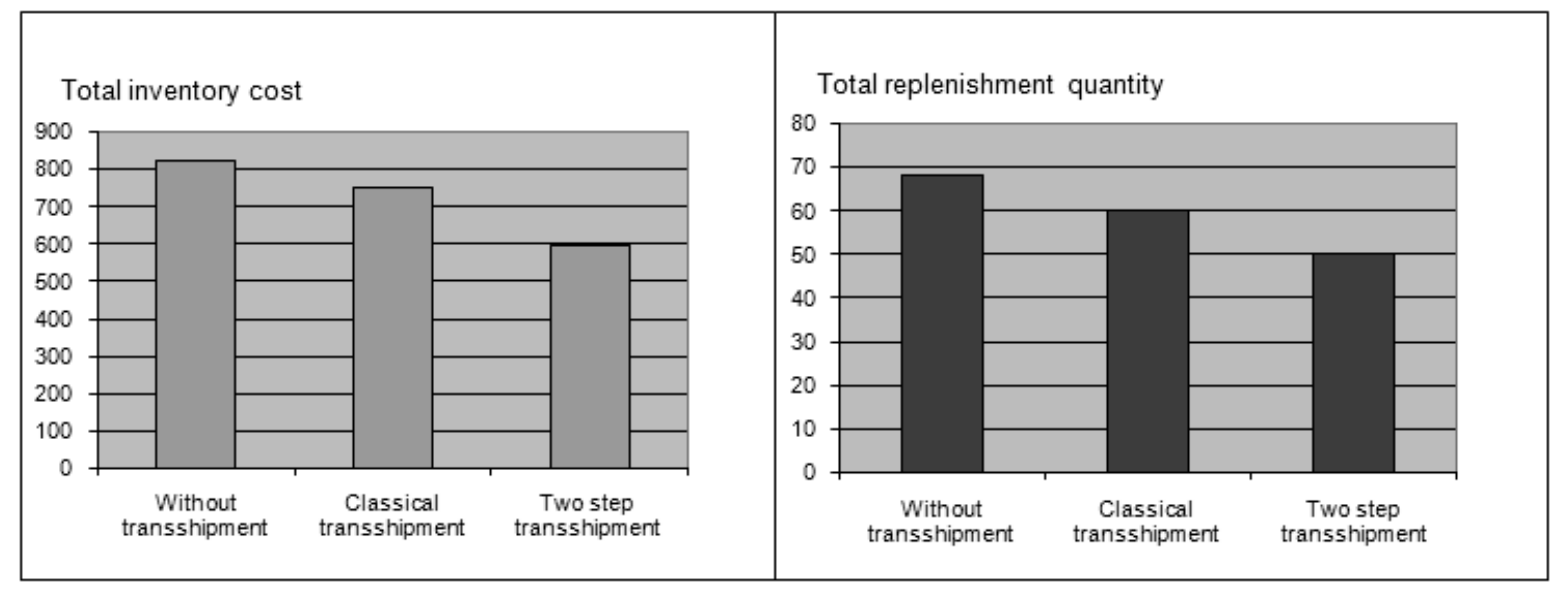

(a)

(b)

Figure 6: Comparing between configurations according to: (a) total inventory cost and (b) total replenishment quantity.

\subsection{The influence of customer demands uncertainty}

The objective of this experimentation is to determine the influence of the customer demands uncertainty on the total inventory cost and the total replenished quantity when our transshipment policy is practiced. We define three scenarios: (1) customer demands having membership degree superior to 0.5, (2) customer demands membership degree is superior to 0.7 and (3) customer demands membership degree is equal to 1 . For this experimentation, we choose also a common service level which is equal to 0.80 .

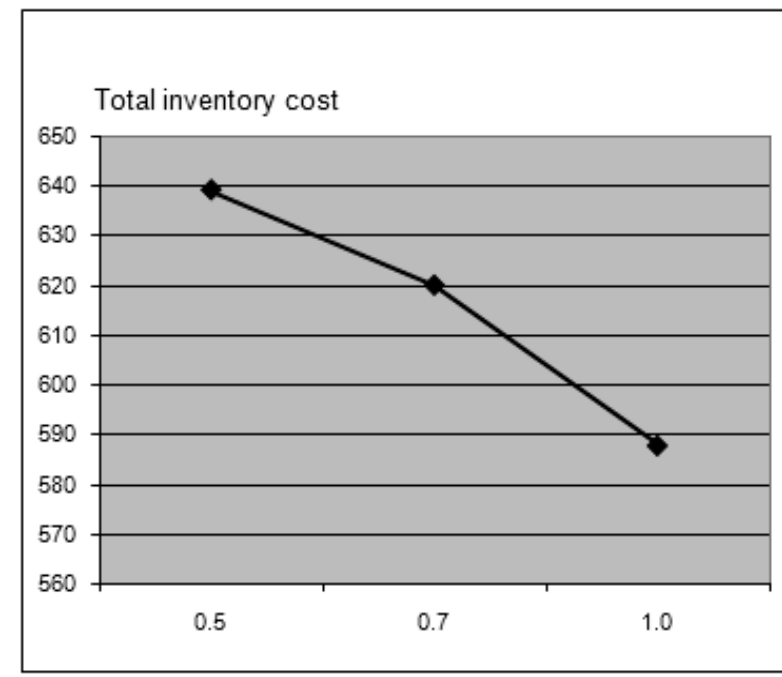

(a)

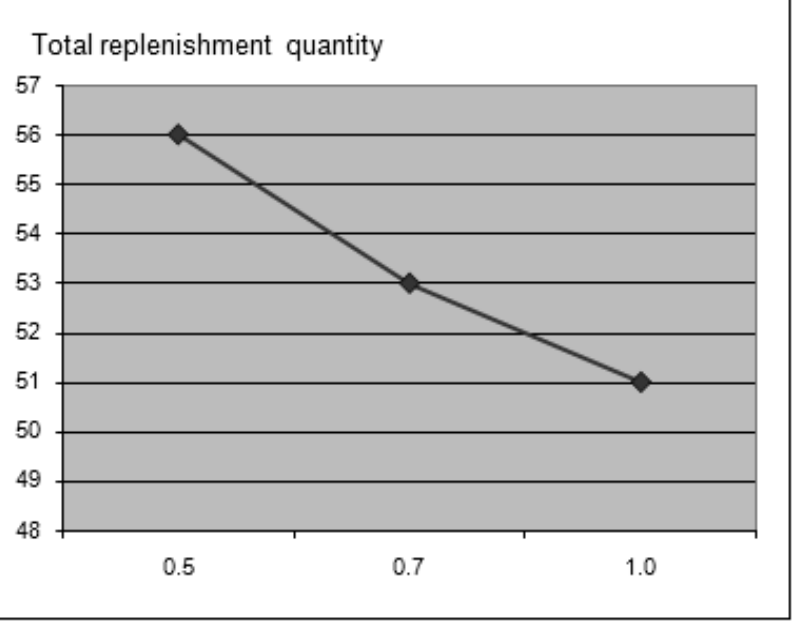

(b)

Figure 7: Comparing scenarios according to: (a) total inventory cost and (b) total replenished quantity.

Fig. 7 (a) shows that the minimum total inventory cost is that of the third scenario having the lowest customer demands uncertainty (membership degree $=1$ ). We remark also that the total inventory cost decreases when the customer demands uncertainty decrease. This could be explained by the fact that when customer demands uncertainty is high, having a reduced membership degree, we need a high level of total replenished quantity to satisfy the service level constraints. This is shown by Fig. 7 (b). 


\subsection{The influence of service level constraints on inventory costs}

In this section our objective is to identify the influences of service level constraints on the total inventory cost. We define the Target global service level (TGSL) criterion given in Eq. (18).

$$
T G S L=\frac{1}{N} \times \sum_{i=1}^{N} \beta_{i}
$$

Fig. 8 provides an overall validation of the service level constraints impact on the inventory cost. In fact, both total inventory cost and total replenishment quantity increase proportionally with the increase of the TGSL as shown respectively in Figs. 8 (a) and 8 (b).

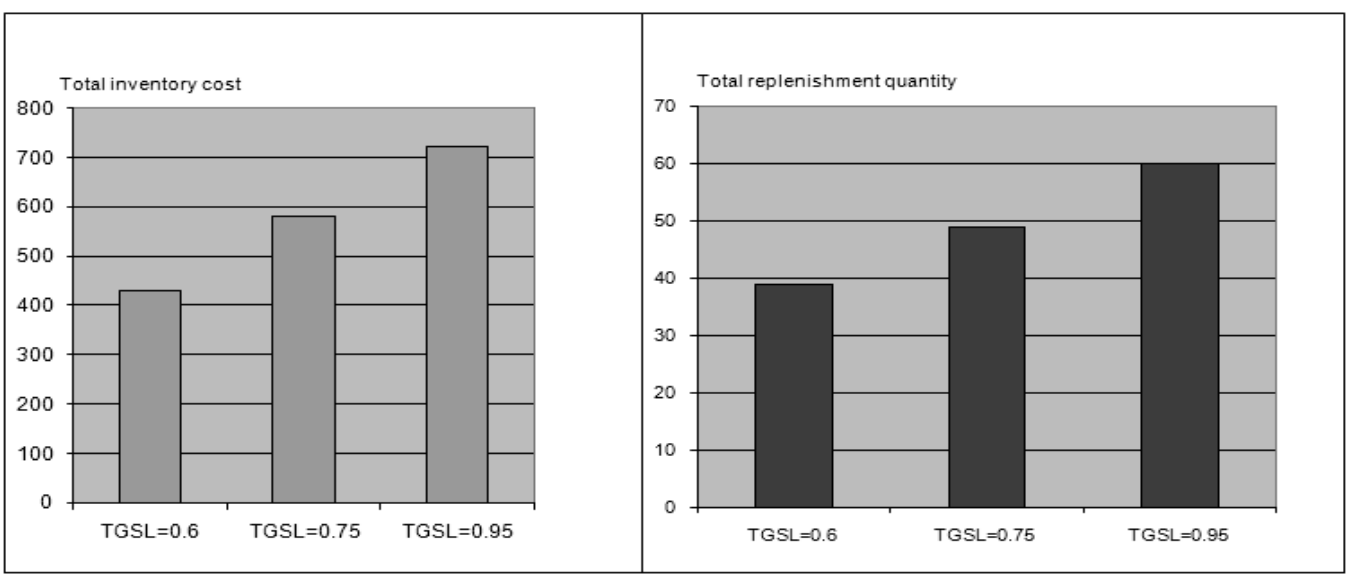

(a)

(b)

Figure 8: Influence of service level constraints on total inventory cost (a) and total replenishment quantity (b).

Table II: Configurations of minimum service level $\beta_{i}$.

\begin{tabular}{|c|c|c|c|c|}
\hline Configuration & $\beta_{1}$ & $\beta_{2}$ & $\beta_{3}$ & $\beta_{4}$ \\
\hline 1 & 0,75 & 0,75 & 0,75 & 0,75 \\
\hline 2 & 0,95 & 0,55 & 0,65 & 0,85 \\
\hline 3 & 0,65 & 0,65 & 0,85 & 0,85 \\
\hline
\end{tabular}

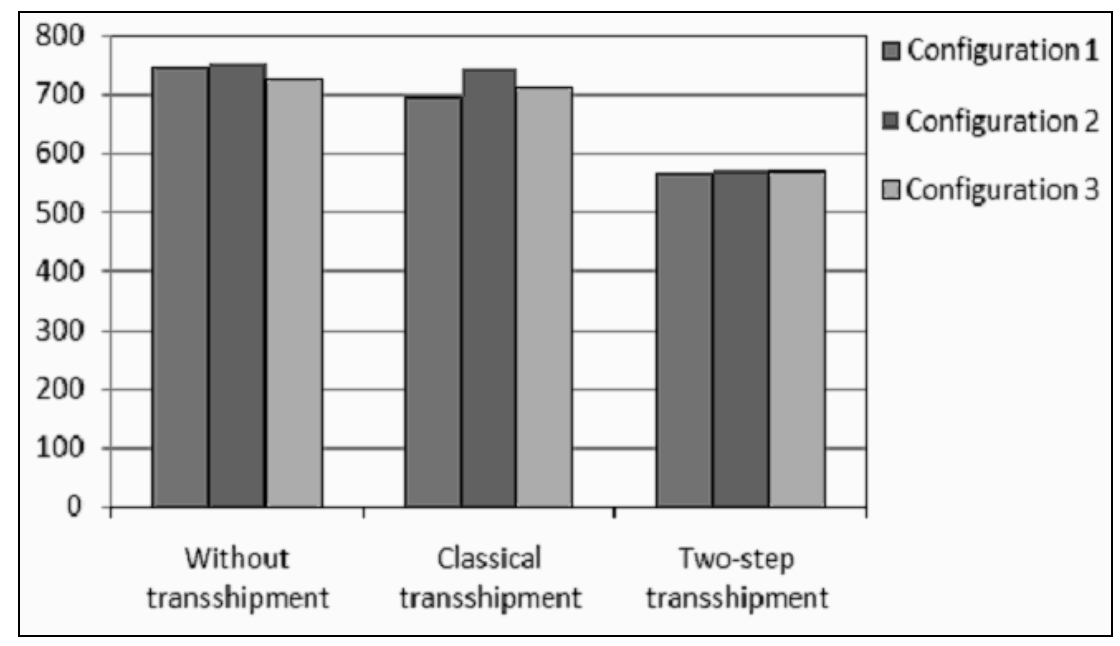

Figure 9: Comparing the three configurations. 
In the following experimentation we consider three different configurations corresponding to $T G S L=0.75$ (Table II). According to result experimentation presented in Fig. 9, we notice that for inventory systems where no transshipment or classical transshipment is practiced that the total inventory cost varies from one configuration to another.

However, for the inventory system where our two-step transshipment policy is practiced, we obtain almost the same total inventory cost. This proves that this transfer policy helps local service level to converge to the global one. In fact, sharing the total available inventory even when there are shortages is a key compound to ensure a high trade-off between local service level constraint and the global inventory cost.

\section{CONCLUSION}

We have considered the transshipment problem characterized by fuzzy customer demands and service level constraints. Our objectives were to identify a transshipment policy that helps to satisfy the service level constraints at minimum inventory costs and to determine the replenishment quantities. We have proposed a two-step transshipment policy: in the first step, the transfer is executed from locations in excess to those that did not achieve their required service level. In the second step, the transfer is realized from locations in need that achieved their required service level to those that did not realize their required service level.

To determine the replenishment quantities, we proposed: (1) a new model of the transshipment problem based on the chance constrained programming and (2) we developed a hybrid algorithm based on fuzzy simulation and genetic algorithm. Our experimental results have shown that: (a) the proposed transshipment policy is better that the classical one characterized by transfer from transshipment in excess to those in need, (b) the total inventory cost increase when the customer demands uncertainty increase and (c) when the two-step transshipment policy is practiced the local required service level converge to the global one. Our future works will consider a fuzzy required service level and fuzzy inventory costs.

\section{REFERENCES}

[1] Fisher, M. (1997). What is the right supply chain for your product?, Harvard Business Review, Vol. 75, 105-116

[2] Krishnan, K. S.; Rao, V. R. K. (1965). Inventory control in N warehouses, Journal of Industrial Engineering, Vol. 16, 212-215

[3] Eppen, G. (1979). Effects of centralization on expected costs in a multi-location newsboy problem, Management Science, Vol. 25, 498-501

[4] Herer, Y. T., Tzur, M., Yucesan, E. (2006). The multilocation transshipment problem, IIE Transactions, Vol. 38, 185-200

[5] Tagaras, G.; Cohen, M. A. (1992). Pooling in two-location inventory systems with non-negligible replenishment lead times, Management science, Vol. 38, No. 8, 1067-1083

[6] Tagaras, G. (1999). Pooling in multi-location periodic inventory distribution systems, International journal of management science, Vol. 27, 39-59

[7] Herer, Y. T., Tzur, M.; Yucesan, E. (2002). Transshipment: An emerging inventory recourse to achieve Supply chain leagility, International journal of production and economics, Vol. 80, 201212

[8] Tagaras, G. (1989). Effects of pooling in two-location inventory systems, IIE Transactions, Vol. 21, 250-257

[9] Herer, Y. T.; Rashit, A. (1999). Lateral stock transshipments in a two-location inventory system with fixed and joint replenishment costs, Naval Research Logistics, Vol. 46, 525-547

[10] Ozdemir, D., Yucesan, E.; Herer, Y. T. (2006). Mutli-location transshipment problem with capacitated transportation, European Journal of Operational Research, Vol. 175, 602-621 
[11] Tagaras, G.; Vlachos, D. (2002). Effectiveness of stock transshipment under various demand distributions and non-negligible transshipment times, Production and Operations Management, Vol. 11, No. 2, 183-198

[12] Robinson, L. W. (1990). Optimal and Approximate in Multiperiod Multilocation Inventory Models with Transshipment, Operations Research, Vol. 38, 278-295

[13] Petrovic, D.; Vujo, M. (1996). Fuzzy models for the newsboy problem, International Journal of Production Economics, Vol. 45, 435-441

[14] Petrovic, D.; Roy, R.; Petrovic, R. (1998). Modelling and simulation of a supply in an uncertain environment, European journal of operational research, Vol. 109, No. 2, 299-309

[15] Dey, J. K., Kar, S.; Maiti, M. (2005). An interactive method for inventory control with fuzzy lead-time and dynamic demand, European Journal of Operational Research, Vol. 167, 381-397

[16] Rong, M., Mahapatra, N. K.; Maiti, M. (2008). A two warehouse inventory model for a deteriorating item with partially/fully backlogged shortage and fuzzy lead time, European Journal of Operational Research, Vol. 189, 59-75

[17] Ishii, H.; Konno, T. (1998). A stochastic inventory problem with fuzzy shortage cost, European Journal of Operational Research, Vol. 106, 90-94

[18] Aardal, K.; Jonsson, O.; Jonsson, H. (1989). Optimal inventory policies with service-level constraints, Journal of the operational research society, Vol. 40, No. 1, 65-73

[19] Chiang, K.; Wen-Kai, H. (2002). A single period inventory model with fuzzy demands, Computers and mathematics with applications, Vol. 43, 841-848

[20] Petrovic, D., Roy, R.; Petrovic, R. (1999). Supply chain modelling using fuzzy sets, International journal of production economics, Vol. 59, 443-453

[21] Roy, T. K.; Maiti, M. (1997). A fuzzy EOQ model with demand-dependent unit cost under limited storage capacity, European Journal of Operational Research, Vol. 99, 425-432

[22] Axsater, S. (2006). A simple procedure for determining order quantities under a fill-rate constraint and normally distributed lead time demand, European journal of operational research, Vol. 174, 480-491

[23] Brian, K.; Katircioglu, K.; Puterman, M. L. (1999). Retail inventory control with lost sales, service constraints and fractional lead times, Production and operations management, Vol. 8, No. 4, 393-408

[24] Chen, F. Y.; Krass, D. (2001). Inventory models with minimal service level constraints, European journal of operational research, Vol. 134, 120-140

[25] Wu, J., Wang, S.; Yue, S. (2005). Supply contract model with service level constraint, Journal of industrial and management optimization, Vol. 1, No. 3, 275-287

[26] Young, Y. L., Jung, J. W.; Young, S. J. (2007). An effective lateral transshipment policy to improve service level in the supply chain, International journal of production economics, Vol. 126, 115-126

[27] Zadeh, L. A. (1965). Fuzzy sets, Information and Control, Vol. 8, 338-353

[28] Liu, B.; Iwamura, K. (1998). Chance constrained programming with fuzzy parameters, Fuzzy Sets and Systems, Vol. 94, 227-237

[29] Holland, J. H. (1975). Adaptation in Natural and Artificial Systems, University of Michigan Press, Ann Arbor 